\title{
ORIGINAL
}

\section{ESTUDIO LONGITUDINAL DE LOS REINGRESOS EN PACIENTES CON INSUFICIENCIA CARDIACA EN CATALUÑA, 1996-1999 (*)} \author{
Lluís Tomàs $(3)$ \\ (1) Universitat Autònoma de Barcelona. Cerdanyola del Vallès \\ (2) Agència d'Avaluació de Tecnologia i Recerca Mèdiques. Barcelona \\ (3) Servei de Cardiologia de l'Hospital de la Santa Creu i Sant Pau. Barcelona
}

Josefina Caminal (1), Eduardo Hermosilla (1), Emilia Sánchez (2), Mireya Urrea (1), Pere Puig (1),

\section{RESUMEN}

Fundamento: El objetivo de este trabajo es describir el fenómeno del reingreso para los pacientes con insuficiencia cardiaca (IC) e identificar algunos de sus factores clínicos asociados, mediante un identificar algunos de sus factores clínicos asocias
estudio de seguimiento con datos administrativos.

Métodos: Estudio longitudinal de reingresos por insuficiencia cardiaca congestiva en la población $\geq 65$ años de Cataluña durante período 1996-1999. Fuent Catalán de la Salud Definición de insuficiencia cardiaca congestiva, causa etiológica o precipitante y comorbilidad mediante la combinación de códigos CIE-9 de alta. Unidades de análisis: 'paciente con insuficiencia cardiaca congestiva' y 'población índice o cohorte' Estadística: análisis de supervivencia (Kaplan-Meier y test de Logrank) y modelos de regresión (Cox).

Resultados: La población de seguimiento integrada por 16.919 pacientes generó 44.283 altas $(61,8 \%$ reingresos). El tiempo libre de reingreso (Mediana 'ME' en meses) y la comparación de las curvas de supervivencia es estadísticamente significativa con valores inferiores en las categorías: '65-74 años' (ME=21,6 meses); 'hombre' (23,3); 'región sanitaria 6' (16,3); 'hospital de referencia' $(22,9)$ y 'EPOC pura asociada a la IC (17,7). El mayor riesgo de reingreso, ajustado por las otras variables de estudio, se observa para 'EPOC pura' $[R R=1,03$, (IC 95\%: 1,02-1,04), p<0,001], y 'Cardiopatía isquémica' [RR=1,03, (IC al 95\%: 1,01-1,05), $\mathrm{p}=0,003]$.

Conclusiones: Este estudio muestra la complejidad clínica y patrones de utilización hospitalaria de los pacientes con insuficiencia cardiaca, identificando que los que padecen EPOC o cardiopatía isquémica constituyen grupos prioritarios de intervención asistencial y mostrando de este modo el potencial de las bases de datos administrativas para la planificación y la gestión clínica.

Palabras clave: Estudios longitudinales. Insuficiencia cardiaca. Reingresos hospitalarios.

\section{Correspondencia:}

Dra. Josefina Caminal-Homar

Universitat Autònoma de Barcelona. Campus de Bellaterra. Edifici M

(08193) Cerdanyola del Vallès

Teléfono: 935813802

Fax: 935812344

Correo electrónico: josefina.caminal@uab.es

\section{ABSTRACT}

\section{Longitudinal Study of Hospital Readmissions for Heart Failure in Catalonia, 1996-1999}

Background: This study is aimed at describing the readmission phenomenon for heart failure patients and identifying some of their related clinical factors by means of a follow-up study with administrative data.

Methods: Longitudinal study of readmissions due to heart failure (HF) among a population $\geq$ age 65 in Catalonia throughout the 19961999 period Information source: Minimum Basic Set of Data of Hospital Discharges from the Catalan Health Service. Definition of HF etiological or precipitating cause, and comorbility by means of a ICD- 9 disharge release codes combination. Analysis units «patient with HF» and an index population or cohort». Statistics: survival analysis (Kaplan-Meier and Log-rank test) and regression models (Cox).

Results: The follow-up populations, comprised of 16,919 patients, generated 44,283 admissions $(61.8 \%)$. The length of time free of readmissions (Mean "ME" in months) and the comparison of the survival curves is statistically significant with lower values in the following categories: 'age 65-74' (ME=21.6 months); 'male' (23.3); 'healthcare region 6' (16.3); 'reference hospital' (22.9) and 'pure COPD related to the HF' (17.7). The highest risk of readmission, adjusted by the other variables under study, has been found for 'pure COPD' [RR=1.03, (95\% CI: 1.02-1.04), p $<0.001$ ], and ischaemic heart disease $[\mathrm{RR}=1.03$, (95\% CI: 1.01-1.05), $\mathrm{p}=0.003]$.

Conclusions: This study reveals the clinical complexity and patterns of utilization of hospitals on the part of patients with heart failure, identifying that those having COPD or ischaemic heart disease being top-priority groups for care intervention and thus revealing the potential which administrative data has for clinical planning and management.

Key words: Longitudinal studies. Heart failure. Hospital readmissions.

(*) Proyecto financiado parcialmente por el Fondo de Investigaciones Sanitarias 02/1306. 


\section{INTRODUCCIÓN}

La insuficiencia cardiaca (IC) se considera como uno de los mayores y más crecientes problemas de salud pública en los países industrializados, con tasas de admisiones hospitalarias que se incrementan progresivamente y se asocian a una elevada frecuencia de reingresos, muchos de ellos considerados potencialmente evitables ${ }^{1-5}$. Una extensa producción científica describe sus características clínico-epidemiológicas ${ }^{6-10}$ y cuantifica la tendencia creciente al consumo de recursos sanitarios ${ }^{7,11}$. Además, la magnitud, complejidad y requerimientos asistenciales de los pacientes afectos de esta dolencia han propiciado el desarrollo de guías de práctica clínica $^{12-14}$ y novedosas iniciativas organizativas para ofertar una atención integral y mejorar su calidad de vida ${ }^{3,15,16}$. El análisis de la situación refleja que la mayoría de los estudios referenciados utilizan información clínica procedente de los archivos de historias clínicas del hospital, pocos son estudios de seguimiento de cohortes y no se ha encontrado ninguno en el ámbito español que plantee el seguimiento de pacientes utilizando datos administrativos.

Sin embargo, el diseño de estudios longitudinales basados en datos administrativos, como el Conjunto Mínimo Básico de Datos de Alta Hospitalaria (CMBD-AH), ha sido propuesto por algunos autores anglosajones como una buena alternativa para incrementar el conocimiento de las enfermedades y sus determinantes ${ }^{17-18}$. Las fortalezas del CMBD-AH y la posibilidad de controlar sus limitaciones validan el uso secundario de estas bases de datos más allá de los objetivos para los que fueron diseñadas ${ }^{19}$. Entre las fortalezas cabe citar su calidad ${ }^{20}$, la exhaustividad de los registros de altas en el tiempo y el territorio, su recogida sistemática y, la homogeneidad de las variables y de la herramienta de codificación ${ }^{19}$. Mientras, con relación a las limitaciones, existen trabajos que han demostrado la factibilidad de control mediante una adecuada aproximación meto- dológica y de manejo ${ }^{21-22}$ posibilitando el enfoque que incorpora la complejidad de la enfermedad en el individuo ${ }^{23}$, propósito primordial de toda investigación en servicios sanitarios.

El objetivo de este trabajo es describir el fenómeno del reingreso para los pacientes con IC e identificar algunos de sus factores clínicos asociados, mediante un estudio de seguimiento con datos administrativos.

\section{MATERIAL Y MÉTODOS}

Estudio longitudinal con datos retrospectivos de los reingresos por IC, o por causa relacionada con la IC, en la población de 65 años y más durante el período 1996-1999 en Cataluña. La fuente de información fue el CMBD-AH del Servei Català de la Salut ${ }^{24}$. Estudio exhaustivo que incluyó todas las altas, primeras y repetidas, generadas por la población residente en Cataluña atendida en los hospitales públicos y privados de dicha Comunidad Autónoma.

Como variables explicativas se incluyeron las características del centro y las sociodemográficas y clínicas de los pacientes: Nivel del hospital; Edad (64-74; 75-84 y $\geq$ 85 años); Sexo; Región sanitaria de residencia del paciente; Patología asociada a la IC (hipertensión arterial; cardiopatía isquémica; valvulopatías; cardiomiopatía; malformaciones congénitas; enfermedad pulmonar obstructiva crónica (EPOC) ['mixta' y 'pura' según se combinara o no con las patologías anteriores]); y Otras comorbilidades' (presencia/ausencia). El porcentaje de reingresos, las estancias y el tiempo libre de reingreso, constituyeron las variables respuesta.

El mapa sanitario de Cataluña está organizado en 8 unidades territoriales denominadas regiones sanitarias. En cuanto a la clasificación de los hospitales, el CMBD-AH distingue entre los hospitales privados y los 
de la red pública que clasifica según nivel de complejidad ('comarcal' o de primer nivel, 'de referencia' o de segundo nivel y 'alta tecnología’ o de tercer nivel).

Un punto importante de la metodología fue el manejo de la base de datos del CMBDAH para establecer los perfiles clínicos y crear las distintas unidades de análisis que requirieron la exploración exhaustiva y el tratamiento de la información clínica y administrativa. Los perfiles clínicos se construyeron de acuerdo a las directrices de las sociedades científicas $^{12-14}$, mediante la combinación de los códigos CIE-9 (Clasificación Internacional de Enfermedades, $9^{\mathrm{a}}$ revisión ${ }^{25}$ de alta hospitalaria en todas las posiciones diagnósticas de alta (diagnósticos principal y secundarios). Estos perfiles incluyeron: 'definición de la IC', identificación de las 'causas etiológicas o precipitantes' (anexo 1) ${ }^{26}$ y 'otras comorbilidades'. Mientras que las unidades de análisis fueron 'paciente con IC' y 'población de seguimiento' que se generaron a partir de la combinación de variables administrativas registradas en la base de datos.

La población de seguimiento estaba integrada por aquellos pacientes cuya primera alta por IC se registró durante 1996 (primer año del período de seguimiento 1996-1999) y no fueron terminales (se excluyeron los casos que fallecieron en el primer ingreso).

El análisis estadístico incluyó la descripción de cada una de las variables respuesta a través de estadísticos de frecuencia (número de casos y porcentaje) para los pacientes, las altas y la proporción de reingresos y estadísticos descriptivos (media, desviación estándar, mediana y percentil 75) para la estancia y el tiempo hasta el primer reingreso. Dadas las características de la variable respuesta 'tiempo hasta el primer reingreso' o 'tiempo al primer reingreso', el análisis utilizó las técnicas estadísticas propias del análisis de la supervivencia: probabilidades de supervivencia con el método Kaplan-Meier y la comparación de curvas de supervivencia mediante el test del Log-rank. Las técnicas del análisis de supervivencia, si bien desde un punto de vista histórico se utilizan para describir tiempos de vida, también pueden emplearse para analizar tiempos de ocurrencia de cualquier acontecimiento o evento. En nuestro caso el evento de interés es el 'primer reingreso', cuyo tiempo de ocurrencia se determina a través de la diferencia entre la fecha de alta del primer ingreso y la fecha de reingreso. Las técnicas del análisis de la supervivencia permiten tener en cuenta los denominados 'datos censurados'. Estos estaban constituidos por los pacientes que entre la fecha de alta y la fecha 31 de diciembre de 1999 (fin del estudio) no presentaron ningún reingreso. El estudio multivariante para identificar el riesgo de reingreso de cada una de las patologías asociadas a la IC se llevó a cabo mediante modelos de regresión y utilizó el modelo de riesgos proporcionales de Cox, ajustando por las variables sociodemográficas, clínicas y de características del hospital. El programa informático utilizado para el análisis estadístico fue el programa SPSS para Windows, versión 11.5.

\section{RESULTADOS}

\section{Descriptiva del fenómeno del reingreso}

La población de seguimiento estuvo constituida por 16.919 pacientes (2.036 fallecieron del total de 18.955 casos que generaron su primer alta en 1996). Esta población a lo largo de 1996-1999 produjo un total de 44.283 altas, de las cuales 27.364 fueron reingresos $(61,8 \%)$. La distribución de altas por paciente se presenta como documento adjunto, destacando la existencia de 1.052 pacientes $(2,1 \%)$ con más de ocho altas (anexo 2).

La población $\geq 75$ años constituyó más de la mitad de la población de estudio $(58,0 \%)$, con un predominio de mujeres $(54,4 \%)$ (tabla 1). 
Tabla 1

Distribución de pacientes, altas, estancias y tiempo libre de reingreso (mediana y prueba log-rank) para cada variable

\begin{tabular}{|c|c|c|c|c|c|c|c|c|c|c|c|}
\hline & & \multicolumn{2}{|c|}{ Pacientes } & \multicolumn{2}{|l|}{ Altas } & \multicolumn{3}{|c|}{ Estancias (días) } & \multicolumn{3}{|c|}{$\begin{array}{l}\text { Tiempo libre de } \\
\text { reingresos }\end{array}$} \\
\hline & & $\mathbf{N}$ & $\%$ & $\mathbf{N}$ & $\%$ reingresos & total & media & ds & P50 & P75 & $\begin{array}{l}\text { Valor } \\
\text { de p }\end{array}$ \\
\hline \multirow[t]{4}{*}{ Edad } & $64-74$ & 7.109 & 42,0 & 20.816 & 65,8 & 218.083 & 10,5 & 11,1 & 21,6 & 3,0 & \multirow{4}{*}{$<0,001$} \\
\hline & $75-84$ & 6.298 & 37,2 & 16.193 & 61,1 & 174.496 & 10,8 & 9,4 & 27,8 & 3,3 & \\
\hline & $\geq 85$ & 3.512 & 20,8 & 7.274 & 51,7 & 79.557 & 10,9 & 8,5 & 43,3 & 4,8 & \\
\hline & Total & 16.919 & 100,0 & 44.283 & 61,8 & 472.136 & 10,7 & 10,0 & 35,6 & 4,2 & \\
\hline \multirow[t]{2}{*}{ Sexo } & hombre & 7.723 & 45,6 & 21.429 & 64,0 & 226.332 & 10,6 & 10,2 & 23,3 & 3,0 & \multirow[t]{2}{*}{$<0,001$} \\
\hline & mujer & 9.196 & 54,4 & 22.854 & 59,8 & 245.804 & 10,8 & 9,9 & 33,1 & 3,9 & \\
\hline \multirow[t]{9}{*}{$\begin{array}{l}\text { Patología } \\
\text { asociada }\end{array}$} & $\begin{array}{c}\text { IC sin } \\
\text { patología } \\
\text { asociada }\end{array}$ & 6.373 & 37,7 & 15.655 & 59,3 & 180.477 & 10,7 & 11,0 & 35,9 & 3,8 & \multirow{9}{*}{$<0,001$} \\
\hline & HTA & 1.705 & 10,1 & 3.935 & 56,7 & 33.950 & 11,5 & 8,3 & 36,4 & 4,9 & \\
\hline & $\mathrm{CI}$ & 2.856 & 16,9 & 7.543 & 62,1 & 74.014 & 9,8 & 9,7 & 26,8 & 3,1 & \\
\hline & Valvulopatías & 1.249 & 7,4 & 3.240 & 61,5 & 30.789 & 9,5 & 10,2 & 25,6 & 3,1 & \\
\hline & $\mathrm{CM}$ & 661 & 3,9 & 1.789 & 63,1 & 20.504 & 11,5 & 11,5 & 27,6 & 3,6 & \\
\hline & $\begin{array}{c}\text { Malformacion } \\
\text { es }\end{array}$ & 38 & 0,2 & 121 & 68,6 & 1.597 & 13,2 & 17,0 & 37,0 & 3,9 & \\
\hline & EPOC pura & 2.096 & 12,4 & 6.385 & 67,2 & 71.798 & 11,2 & 9,4 & 17,7 & 2,9 & \\
\hline & EPOC mixta & 569 & 3,4 & 1.534 & 62,9 & 13.566 & 8,8 & 7,4 & 24,7 & 4,1 & \\
\hline & Otras & 1.372 & 8,1 & 4.081 & 66,4 & 45.4411 & 11,1 & 8,2 & 17,9 & 2,4 & \\
\hline \multirow{2}{*}{$\begin{array}{l}\text { Otras } \\
\text { comorbi- } \\
\text { lidades } \\
\text { asociadas }\end{array}$} & no & 4.438 & 26,2 & 11.459 & 61,3 & 105.797 & 9,2 & 10,4 & 30,8 & 3,8 & \multirow[b]{2}{*}{0,213} \\
\hline & sí & 12.481 & 73,8 & 32.824 & 62,0 & 366.339 & 11,2 & 9,9 & 27,2 & 3,2 & \\
\hline \multirow{8}{*}{$\begin{array}{l}\text { Región } \\
\text { sanitaria }\end{array}$} & 1 & 757 & 4,5 & 1.747 & 56,7 & 19,307 & 11,1 & 8,9 & 41,1 & 4,8 & \multirow{8}{*}{$<0,001$} \\
\hline & 2 & 1.031 & 6,1 & 2.598 & 60,3 & 26.960 & 10,4 & 9,4 & 27,2 & 4,5 & \\
\hline & 3 & 411 & 2,4 & 1.085 & 62,1 & 8.701 & 8,0 & 9,1 & 24,1 & 3,5 & \\
\hline & 4 & 1.389 & 8,2 & 3.375 & 58,8 & 30.626 & 9,1 & 8,2 & 35,4 & 3,8 & \\
\hline & 5 & 2.367 & 14,0 & 5.987 & 60,5 & 71.834 & 12,0 & 11,6 & 27,7 & 4,0 & \\
\hline & 6 & 1.616 & 9,6 & 4.818 & 66,5 & 51.200 & 10,6 & 9,6 & 16,3 & 2,3 & \\
\hline & 7 & 3.807 & 22,5 & 10.958 & 65,3 & 115.974 & 10,6 & 9,9 & 19,3 & 2,6 & \\
\hline & 8 & 5.541 & 32,8 & 13.715 & 59,6 & 147.534 & 10,8 & 10,2 & 38,3 & 3,9 & \\
\hline \multirow{4}{*}{$\begin{array}{l}\text { Nivel de } \\
\text { hospital }\end{array}$} & 1 & 5.093 & 30,1 & 12.623 & 59,7 & 122.798 & 9,7 & 8,4 & 35,4 & 4,1 & \multirow{4}{*}{$<0,001$} \\
\hline & 2 & 7.010 & 41,4 & 19.390 & 63,8 & 209.978 & 10,8 & 9,6 & 22,9 & 2,9 & \\
\hline & 3 & 4.134 & 24,4 & 10.734 & 61,5 & 123.258 & 11,5 & 12,2 & 27,7 & 3,3 & \\
\hline & privado & 682 & 4,0 & 1.536 & 55,6 & 16.102 & 10,5 & 10,3 & 45,7 & 5,1 & \\
\hline
\end{tabular}

\$: Prueba log-Rank para la comparación de curvas; IC: insuficiencia caedíaca; HTA: hipertensión arterial; CI: Cardiopatía isquémica; CM: Cardiomiopatía; EPOC: Enfermedad pulmonar obstructiva crónica.

La utilización de recursos medidos en estancias hospitalarias registró un total de 472.136 días de hospitalización. Las variaciones de la estancia media fueron más manifiestas para la patología asociada y entre regiones sanitarias, distinguiéndose un rango de 8,6 a 13,2 días por alta y de 8,0 a 12,0 , respectivamente (tabla 1 ).

\section{Análisis del tiempo libre de ingreso: análisis de la supervivencia}

La comparación de las curvas de supervivencia mediante el test del Log-rank muestra diferencias estadísticamente significativas ( $p<0,001)$ entre las categorías de las siguientes variables estudiadas, observándose el menor tiempo hasta el primer reingreso para la categoría señalada: Edad (65-74 [mediana 'ME' del tiempo hasta el primer reingreso en meses $\mathrm{ME}=21,6$ meses], 75-84 y 85 años); Sexo (mujer y hombre [ME=23,3]); Región sanitaria (RS) (de las 8 RS, la RS 6 $[\mathrm{ME}=16,3]$ ); Nivel hospitalario (comarcal, de referencia $[\mathrm{ME}=22,9]$, alta tecnología) y Patología relacionada con la IC (IC sin especificación de patología asociada, hipertensión arterial, cardiopatía isquémica, valvulopatía, miocardiopatía, malformaciones 
Tabla 2

Análisis multivariante de Cox

\begin{tabular}{|l|c|c|c|c|}
\hline \multirow{2}{*}{ Patología asociada } & & \multicolumn{2}{l|}{ Intervalo de confianza (límites) } & \multicolumn{1}{c|}{} \\
\cline { 2 - 5 } & $\mathbf{R R}$ & Inferior & Superior & Valor de p \\
\hline IC sin patología asociada & 1 & & & \\
\hline Hipertensión arterial & 0,97 & 0,94 & 1,01 & 0,120 \\
\hline Cardiopatía isquémica & 1,03 & 1,01 & 1,05 & 0,003 \\
\hline Valvulopatías & 1,01 & 0,99 & 1,03 & 0,630 \\
\hline Cardiomiopatía & 0,99 & 0,97 & 1,01 & 0,220 \\
\hline Malformaciones & 0,99 & 0,92 & 1,06 & 0,750 \\
\hline E pulmonar obstructiva crónica pura & 1,03 & 1,02 & 1,04 & $<0,001$ \\
\hline E pulmonar obstructiva crónica mixta & 1,00 & 0,98 & 1,02 & 0,950 \\
\hline Otras combinaciones & 1,01 & 1,00 & 1,02 & 0,030 \\
\hline
\end{tabular}

\$: Modelo ajustado por edad sexo, región sanitaria del paciente, nivel del hospital y otras morbilidades.

congénitas, EPOC pura [ME=17,7], EPOC mixta y combinaciones de patologías asociadas); con excepción de la variable comorbilidades (sí/no [p=0,213]) (tabla 1). El análisis de la supervivencia podría realizarse no sólo para la 'cohorte', sino también para la población total, pero esto estaba fuera de los objetivos de este estudio.

\section{Análisis multivariante: modelo de riesgos proporcionales de Cox}

El mayor riesgo de reingreso, ajustando por las otras variables de estudio (edad, sexo, región sanitaria del paciente, nivel del hospital y presencia de otras comorbilidades), es para los pacientes con IC asociada a la EPOC pura $[R R=1,03$ IC95\% $(1,02-1,04)$, $\mathrm{p}<0,001]$ y a la cardiopatía isquémica [RR=1,03 IC95\% (1,01-1,05), p=0,003], seguido por los pacientes con IC asociada a combinaciones de patologías $[\mathrm{RR}=1,01$, IC95\% $(1,00-1,02) \mathrm{p}=0,030]$ (tabla 2).

\section{DISCUSIÓN}

La identificación del paciente, la caracterización clínica de la IC como síndrome complejo y la cuantificación del fenómeno del 'reingreso' a partir de datos adminis- trativos, constituyen las aportaciones más importantes de este trabajo que carece de precedentes en el ámbito nacional.

Habitualmente, los estudios con datos del CMBD-AH utilizan la unidad de análisis 'alta' y analizan solamente la causa registrada y codificada en el diagnóstico principal que ha motivado la hospitalización. Este trabajo a partir de la unidad 'alta' generó la variable 'paciente con IC' mediante la secuencia de todas las altas por IC en cualquier posición diagnóstica, atribuibles al mismo individuo en el mismo hospital. La disponibilidad de la información clínica de la IC registrada en los diagnósticos secundarios se sustenta en la gravedad y características de esta enfermedad por representar un riesgo añadido para otras enfermedades ${ }^{10,27-30}$. Sin embargo, debido a la inexistencia de un identificador que permita fusionar la información de todos los hospitales, podría existir una cierta sobreestimación de pacientes atribuible a posibles duplicidades de aquellos que utilizan más de un centro. Este sesgo quedará resuelto cuando el CMBD-AH incorpore el código de identificación personal. Mientras, a efectos de este estudio, estas duplicidades se controlaron mediante el factor de corrección obtenido de la variable 'traslado a otro hospital'. 
En cuanto a la selección de la 'población de seguimiento', la limitación más importante radica en la arbitrariedad del criterio de elegibilidad debido a que determinados pacientes pueden tener una historia previa de ingresos hospitalarios. Por ello, la selección de los sujetos no es uniforme ${ }^{31}$, en particular, en el caso de la población de este trabajo, los $\geq 65$ años, en la que la enfermedad puede estar ya instaurada. Sin embargo, las limitaciones referidas a la selección de los individuos, no alteran substancialmente los resultados porque el objetivo de este trabajo no es de tipo epidemiológico sino exploratorio.

La caracterización clínica de la IC hace referencia tanto a la etiología o enfermedades precipitantes como a la interrelación con otras comorbilidades ${ }^{27-29}$. Sin embargo, la disponibilidad de solamente 4 diagnósticos para este trabajo, limitó la construcción de perfiles clínicos complejos, de manera que la variable 'otras comorbilidades' se trató simplemente como presencia/ausencia.

Otra precisión hace referencia al enfoque del fenómeno del reingreso al no coincidir con el uso que habitualmente se le da como indicador de calidad de la actividad hospitalaria ${ }^{32}$. Este estudio no tuvo en cuenta ni el tiempo de ocurrencia del reingreso ni si la causa que lo generó era la misma o relacionada con el ingreso anterior ${ }^{33}$ porque el objetivo de este trabajo era identificar y caracterizar a los pacientes con riesgo de reingresar y cuantificar el volumen de actividad hospitalaria.

Desde el punto de vista de la cuantificación de los reingresos, el diseño longitudinal aporta más información que la que ofrece la medida convencional de este fenómeno como media anual de altas de repetición. Estudios en los Estados Unidos informan de tasas de reingresos por IC que varían ampliamente según la población a estudio, metodología utilizada y duración del periodo de observación y varían entre 11-14\% a los 14 días y 36-44\% a los 180 días $^{1,4-5}$. En España hasta 1996 no existían publicaciones sobre reingresos hospitalarios por IC, el primer trabajo analizó la proporción de reingresos urgentes por un diagnóstico relacionado en 30 días desde el alta hospitalaria y lo cuantificó en $13,6 \%{ }^{34}$.

Otra aportación de este trabajo es la identificación de importantes márgenes de variabilidad entre regiones sanitarias que podrían reflejar desigualdad desde el punto de vista de la salud ${ }^{35}$. Las variaciones detectadas hacen referencia al volumen de reingresos, la relación de éstos con la duración de la estancia y el tiempo hasta el primer reingreso. A pesar del interés que tiene el análisis de las causas de las variaciones en la práctica médica, la multiplicidad de factores implicados lo convierte en un tema controvertido requiriendo estudios específicos que exceden los objetivos de este trabajo.

El efecto de la patología asociada a la IC sobre el tiempo hasta el primer reingreso puede valorarse mucho mejor en el modelo de regresión de riesgos proporcionales de Cox que en el modelo simple. En él se observa que los pacientes con EPOC pura (es decir, no asociada a otras patologías relacionadas con la IC) o con cardiopatía isquémica son los pacientes que tienen mayor riesgo de reingreso. Esta población constituye la población diana de programas de prevención secundaria, deseable tanto para retardar la aparición de la IC como para prestar una atención más compleja cuando ésta ya se ha desarrollado.

En conclusión, este estudio muestra la complejidad clínica y patrones de utilización hospitalaria de los pacientes con IC, identificando que los que padecen EPOC o cardiopatía isquémica constituyen grupos prioritarios de intervención asistencial y mostrando de este modo el potencial de las bases de datos administrativas para la planificación y la gestión clínica. 
Anexo 1

Códigos CIE-9 para definir la insuficiencia cardiaca y las patologías etiológicas o precipitantes

\begin{tabular}{|l|l|}
\hline \multicolumn{1}{|c|}{$\begin{array}{c}\text { Denominación de las patologías } \\
\text { (categorías establecidas para analizar la complejidad de la } \\
\text { enfermedad) }\end{array}$} & $\begin{array}{l}\text { Códigos CIE-9-MC } \\
\text { (en cualquier posición diagnóstica) }\end{array}$ \\
\hline Insuficiencia cardiaca (IC) & $402.01 ; 402.11 ; 402.91 ; 428 ; 518.4$ \\
\hline Patologías etiológicas o precipitantes de la IC (categorías): & \\
\hline IC sin patología asociada & solamente se registra IC \\
\hline Hipertensión arterial (HTA) & $401 ; 402.00 ; 402.10 ; 402.90$ \\
\hline Cardiopatía isquémica (CI) & $410-414$ \\
\hline Valvulopatías & $394-397 ; 424$ \\
\hline Miocardiopatías & 425 \\
\hline Malformaciones congénitas & $745-747$ \\
\hline Enfermedad pulmonar obstructiva crónica & \\
\hline (1) EPOC pura & $(1) 490-492 ; 496$ \\
\hline (2) EPOC mixta & $\begin{array}{l}\text { (2) combinación de la EPOC con alguna de las patologías } \\
\text { cardiovasculares anteriores }\end{array}$ \\
\hline Otras combinaciones & $\begin{array}{l}\text { presencia de dos o más de las patologías anteriores } \\
\text { asociadas a IC }\end{array}$ \\
\hline
\end{tabular}


Anexo 2

Distribución del número de altas por paciente

\begin{tabular}{|c|c|c|c|}
\hline & Frecuencia & $\%$ & $\%$ acumulado \\
\hline 1 & 33.739 & 45,126 & 45,126 \\
\hline 2 & 17.457 & 23,349 & 68,474 \\
\hline 3 & 9.687 & 12,956 & 81,430 \\
\hline 4 & 5.389 & 7,208 & 88,638 \\
\hline 5 & 3.176 & 4,248 & 92,886 \\
\hline 6 & 1.859 & 2,486 & 95,372 \\
\hline 7 & 1.152 & 1,541 & 96,913 \\
\hline 8 & 722 & 0,966 & 97,879 \\
\hline 9 & 463 & 0,619 & 98,498 \\
\hline 10 & 354 & 0,473 & 98,971 \\
\hline 11 & 206 & 0,276 & 99,247 \\
\hline 12 & 152 & 0,203 & 99,450 \\
\hline 13 & 96 & 0,128 & 99,579 \\
\hline 14 & 88 & 0,118 & 99,696 \\
\hline 15 & 48 & 0,064 & 99,761 \\
\hline 16 & 38 & 0,051 & 99,811 \\
\hline 17 & 31 & 0,041 & 99,853 \\
\hline 18 & 22 & 0,029 & 99,882 \\
\hline 19 & 24 & 0,032 & 99,914 \\
\hline 20 & 8 & 0,011 & 99,925 \\
\hline 21 & 8 & 0,011 & 99,936 \\
\hline 22 & 8 & 0,011 & 99,947 \\
\hline 23 & 6 & 0,008 & 99,955 \\
\hline 24 & 3 & 0,004 & 99,959 \\
\hline 25 & 2 & 0,003 & 99,961 \\
\hline 26 & 7 & 0,009 & 99,971 \\
\hline 27 & 9 & 0,012 & 99,983 \\
\hline 28 & 1 & 0,001 & 99,984 \\
\hline 29 & 2 & 0,003 & 99,987 \\
\hline 30 & 2 & 0,003 & 99,989 \\
\hline 31 & 2 & 0,003 & 99,992 \\
\hline 34 & 1 & 0,001 & 99,993 \\
\hline 36 & 1 & 0,001 & 99,995 \\
\hline 37 & 1 & 0,001 & 99,996 \\
\hline 39 & 1 & 0,001 & 99,997 \\
\hline 40 & 1 & 0,001 & 99,999 \\
\hline 58 & 1 & 0,001 & 100 \\
\hline Total & 74.767 & 100 & \\
\hline
\end{tabular}




\section{BIBLIOGRAFÍA}

1. Gooding J, Jette AM. Hospital readmissions among the elderly. J Am Geriatric Soc 1985; 33: 595-601.

2. Vinson JM, Rich MW, Sperry JC, McNamara T. Early readmission of elderly patients with congestive heart failure. J Am Geriatric Soc 1990; 38: 1290-5.

3. Rich MW, Beckham V, Leven CL, Freedland KE, Carney RM. A multidisciplinary intervention to prevent the readmission of elderly patients with congestive heart failure. N Engl J Med 1995; 333 : 1190-95.

4. Kumholz HM, Parent EM, Vaccarino V, Radford MJ, Hennen J. Readmission after hospitalization for congestive heart failure among Medicare beneficiaries. Arch Intern Med 1997; 157:99-104.

5. Michalsen A, König G, Thimme W. Preventable causative factors leading to hospital admission with decompensated heart failure. Heart 1998; 80: 437-41.

6. Opasich C, Febo O, Riccardi G, Traversa E, Forni G, Pinna G, et al. Concomitant factors of decompensation in chronic heart failure. Am J Cardiol 1996; 78: 354-57.

7. Rodríguez-Artalejo F, Guallar-Castillón P, Banegas JR, del Rey J. Trends in hospitalization and mortality for heart failure in Spain, 1980-1993. Eur Heart J 1997; 18:1771-9.

8. Cowie MR, Mosterd A, Wood DA, Deckers JW, Sutton GC, Grobbee DE. The epidemiology of heart failure. Eur Heart J 1997; 18: 208-23.

9. Sáez T, Suárez C, Blanco F, Gabriel R. Epidemiología de las enfermedades cardiovasculares en la población anciana española. Rev Clin Cardiol 1998; 51: 864-73.

10. Rich MW. Clinics in geriatric medicine. Heart failure in the elderly 2000. Clinics in Geriatric Medicine; volume 16, number 3. Philadelphia: WB Saunders Company. A Harcourt Health Sciences Company; 2000.

11. Castellà X, Mompart A, Pérez G. La utilización de los hospitales de agudos por los ancianos. Cataluña, 1982-1990. Gac Sanit 1997; 11: 259-65.

12. Sociedad Española de Medicina Familiar y Comunitaria: Insuficiencia cardiaca congestiva. Recomendaciones. Barcelona: Sociedad Española de Medicina Familiar y Comunitaria; 1997.

13. Navarro-López F, de Teresa E, López-Sendón JL, Castro-Beiras A. Guías del diagnóstico, clasifica- ción y tratamiento de la insuficiencia cardíaca y del shock cardiogénico. Informe del Grupo de Trabajo de Insuficiencia Cardiaca de la Sociedad Española de Cardiología. Rev Esp Cardiol 1999; 52 (supl 2): $1-54$.

14. Guidelines for the diagnosis and treatment of chronic heart failure. Task Force for the diagnosis and treatment of chronic heart failure. Heart Failure, European Society of Cardiology: W. J. Reme and K. Swedberg (Co-Chairmen). Eur Heart J 2001; 22 : 1527-60.

15. Kostis J, Rosen RC, Cosgrove N, Shindler DM, Wilson A. Non pharmacology therapy improves functional and emotional status in congestive heart failure. Chest 1994; 106: 996-1001.

16. Riegel B, Carlson B, Glaser D, Hoagland P. Which patients with heart failure respond best to multidisciplinary disease management? J Card Fail 2000; 6(4):290-99.

17. Evans R, Barer M, Marmor T. Why some people are healthy and other not? The determinants of health of populations. New York: Aldine de Gruyter; 1994.

18. Shi L, Samuels ME, Pease M, Bailey WP, Corley EH. Patient characteristics associated with hospitalisations for ambulatory acre sensitive conditions in South Carolina. South Medical J 1999; 92 (10); 989-98.

19. Caminal J, Silvestre F. Actividad hospitalaria, pacientes y atención primaria. Rev Calidad Asistencial 2003; 18 (2): 164-70.

20. García M, García C. Análisis exhaustivo y de la calidad de la codificación de las altas de un hospital comarcal (1995-1997). Todo Hosp 1999;161:74750 .

21. Halliday ML, Corey PN, Coates RA, Rankin JG. A method for estimating «persons» versus «cases» from hospital morbidity data in the absence of unique personal identifiers. A J of Epidemiol 1987; 125 (5): 885-91.

22. Starfield B. New paradigms for quality in primary care. British Journal of General Practice 2001; 51: 303-9.

23. Caminal J. La calidad en la atención primaria, un reto para el sistema de salud. Gaceta Clínica y Sanitaria 2002; 4 (4): 121.

24. Altes dels hospitals d'aguts de Catalunya 19961999. Informe del registre del conjunt mínim bàsic de dades d'alta hospitalaria (CMBDAH). Generalitat de Catalunya. Barcelona: Departament de Sanitat i Seguretat Social; 2001. 
25. Ministerio de Sanidad y Consumo. Clasificación Internacional de Enfermedades, $9^{a}$ revisión, Modificación Clínica. 4a edición. Ministerio de Sanidad y Consumo: Madrid, 1999.

26. Urrea M, Caminal J, Martín M. Uso de datos administrativos para identificar perfiles clínicos: una aplicación práctica para la insuficiencia cardiaca. Papeles Médicos 2002; 11 (4): 147-152.

27. Charlson ME, Pompei P, Ales KL, MacKenzie CR. A new method of classifying prognostic comorbidity in longitudinal studies; development and validation. J Chronic Dis 1987; 40 (5): 373-83.

28. Elixhauser A, Steiner Cl, Harris R, Coffey R. Comorbidity measures for use with administrative data. Med Care 1998; 36(1): 8-27.

29. Librero J, Peiró S, Ordiñana R. Comorbilidad crónica y homogeneidad de los grupos de diagnósticos relacionados. Gac Sanit 1999; 13: 292-302.

30. Western GP, Satariano WA, Schellevis FG, Van der Bos GAM. Patterns of comorbidity and the use of health services in the Dutch population. Eur J
Public Health 2001;11(4):365-72. [Comentario al artículo por Caminal J. La comorbilidad como variable de medida de necesidad y utilización de servicios sanitarios. Gaceta Clínica y Sanitaria 2001; 4: 85].

31. Delgado M. Estudios de cohortes. UD 4. En M. Delgado y J. Llorca (Eds.), Metodología de la investigación sanitaria. Barcelona: Signo; 2003.

32. Martínez EA, Aranaz J. ¿Existe relación entre el reingreso hospitalario y la calidad asistencial?. Rev Calidad Asistencial 2002; 17: 79-86.

33. Nuin MA, Saldaña ML, de Pedro MT. ¿Nos informan los reingresos de la calidad de atención hospitalaria?. Rev Calidad Asistencial 2002; 17: 67-78.

34. Jiménez A., García J., González E., Lara A., Nogueiras L. Variables predictivas del reingreso hospitalario precoz en la insuficiencia cardiaca. Rev Clin Esp 1998; 198: 799-804.

35. Meneu R. Variabilidad de las decisiones médicas y su repercusión sobre las poblaciones. Barcelona: Masson; 2002. 